\title{
NOTE ON A THEOREM OF E. A. ROBINSON ON SUMS OF STATIONARY RANDOM VARIABLES
}

\author{
BERNARD W. ROOS
}

In a recent paper, E. A. Robinson [1] established necessary and sufficient conditions for the boundedness of the variance of sums of stationary random variables. In a highly elementary fashion, his first condition may be generalized to encompass continuous stochastic processes and sums and integrals of the form $\sum c(n) X(n)$ and $\int_{0}^{t} c(s) X(s) d s$, respectively.

Let $(\Omega, S, P)$ be a probability space, i.e., $P$ is a normed measure on a $\sigma$-algebra $S$ of subsets of a set $\Omega$. Furthermore, let $M$ be the closed linear manifold generated by random variables $X$, which are defined on $\boldsymbol{S}$ and whose squares are integrable. A stochastic process $\{X(u),-\infty<u<\infty\}$ is called stationary in the wide sense if $E X(u+v) \bar{X}(v)=R(u)$ does not depend on $v ; u$ may be either a continuous or discrete parameter. The spectral representation of $X(u)$ is given by [2]

$$
X(u)=\int_{a}^{b} \exp [2 \pi i u \lambda] d Y(\lambda),
$$

where $a=-\infty, b=+\infty$ for continuous parameter processes, $a=-.5$, $b=.5$ for discrete parameter processes. $Y(\lambda)$ is a uniquely determined process with orthogonal increments, $E|d Y(\lambda)|^{2}=d F(\lambda)$, and $F(\lambda)$ is a bounded nondecreasing function, the spectral distribution function of the process. Without loss of generality, it will furthermore be assumed that $E X=0$.

THEOREM. Let $\{X(u),-\infty<u<\infty\}$ be a wide-sense stationary stochastic process with spectral distribution function $F(\lambda)$. For Variance $\left[\int_{0}^{3} c(t) X(t) d t\right]$ or Variance [ $\left.\sum_{n-1}^{k} c(n) X(n)\right]$ to be bounded for all positive values of $s$ or $k$, a necessary and sufficient condition is

$$
\int_{a}^{b}|C(\lambda)|^{2} d F(\lambda)<\infty
$$

where, ${ }^{1}$ for continuous parameter processes $a=-\infty, b=\infty$,

Received by the editors November 12, 1960.

${ }^{1}$ For limits of this type, reference is made to: N. Wiener, The Fourier integral, Cambridge, Cambridge University Press, 1932. 


$$
|C(\lambda)|^{2}=\lim _{r \rightarrow \infty} \frac{1}{T} \int_{0}^{T}\left[\int_{0}^{8} \int_{0}^{s} \exp [2 \pi i \lambda(t-r)] c(t) \bar{c}(r) d t d r\right] d s
$$

and for discrete parameter processes $a=-.5, b=.5$,

$$
|C(\lambda)|^{2}=\lim _{N \rightarrow \infty} \frac{1}{N} \sum_{k=1}^{N}\left[\sum_{n=1}^{k} \sum_{m=1}^{k} \exp [2 \pi i \lambda(n-m)] c(n) \bar{c}(m)\right] .
$$

Proof. The proof is immediate, and since it follows closely that of Robinson for the discrete parameter case, only the proof for continuous parameter processes will be outlined here. The direct part of the proof reads as follows. Let $K$ be an arbitrary positive constant, and let

$$
\begin{aligned}
\text { Variance }\left[\int_{0}^{s} c(t) X(t) d t\right] & =E\left|\int_{0}^{s} c(t) X(t) d t\right|^{2} \\
& =\int_{0}^{8} \int_{0}^{8} R(t-r) c(t) \bar{c}(r) d t d r<K .
\end{aligned}
$$

Interchange of the order of integration in the iterated integrals is justified [2, Chapter IX, §2]. Integrating from 0 to $T$ and taking the limit for $T \rightarrow \infty$ yields

$$
\lim _{T \rightarrow \infty} \frac{1}{T} \int_{0}^{T}\left[\int_{0}^{s} \int_{0}^{8} R(t-r) c(t) \bar{c}(r) d t d r\right] d s<K
$$

but

$$
\lim _{T \rightarrow \infty} \frac{1}{T} \int_{0}^{T}\left[\int_{0}^{s} \int_{0}^{s} R(t-r) c(t) \bar{c}(r) d t d r\right] d s=\int_{-\infty}^{\infty}|C(\lambda)|^{2} d F(\lambda) .
$$

The indirect part of the proof is obtained by reversing the argument.

The following results may be obtained.

1. $c(n) \equiv 1$ yields the condition of Robinson, since

$$
\begin{aligned}
\lim _{N \rightarrow \infty} \frac{1}{N} \sum_{k=1}^{N}\left[\sum_{n=1}^{k} \sum_{m=1}^{k} \exp [2 \pi i \lambda(n-m)]\right] \\
=\lim _{N \rightarrow \infty} \frac{1}{N} \sum_{k=1}^{N}\left|\frac{1-\exp [2 \pi i \lambda k]}{1-\exp [2 \pi i \lambda]}\right|^{2}=\frac{1}{2 \sin ^{2} \pi \lambda} .
\end{aligned}
$$

Consequently, $\int_{-.5}^{.5} d F(\lambda) / \sin ^{2} \pi \lambda<\infty$. For the continuous parameter case one finds $\int_{-\infty}^{\infty} d F(\lambda) / \lambda^{2}<\infty$.

2. $c(t)=\exp [-2 \pi i \mu t]$ yields $\int_{-\infty}^{\infty} d F(\lambda) /\left(\mu^{2}-\lambda^{2}\right)<\infty, \quad c(n)$ $=\exp [-2 \pi i \mu n]$ yields $\int_{-.5}^{.5} d F(\lambda) / \sin ^{2} \pi(\mu-\lambda)<\infty$. 


\section{REFERENCES}

1. E. A. Robinson, Sums of stationary random variables, Proc. Amer. Math. Soc. vol. 11 (1960) pp. 77-79.

2. J. L. Doob, Stochastic processes, New York, Wiley, 1953.

John Jay Hopkins Laboratory for Pure and Applied Science, General Atomic Division of General Dynamics Corporation, San Diego, California

\section{APPROXIMATION OF INTEGRAL CURRENTS BY CYCLES ${ }^{1}$}

\section{HERBERT FEDERER}

The purpose of this note is to prove the following theorem concerning isoperimetric ratios and cyclic approximation:

If $A$ and $B$ are compact Lipschitz neighborhood retracts in $R^{n}$, with $A \supset B$, and $k$ is a positive integer then, there exists a positive number $\rho$ with the following two properties:

(1) For each $X \in \partial I_{k+1}(A)+I_{k}(B)$ there exists a $Y \in I_{k+1}(A)$ such that $\operatorname{spt}(X-\partial Y) \subset B$ and

$$
M(Y)^{k /(k+1)} \leqq \rho\|X\|(A-B) .
$$

(2) For each $T \in I_{k+1}(A)$ there exists an $S \in I_{k+1}(A)$ such that spt $\partial S$ $\subset B$ and

$$
M(T-S)^{k /(k+1)} \leqq \rho\|\partial T\|(A-B) .
$$

The notation is the same as in Normal and integral currents by H. Federer and W. H. Fleming, Ann. of Math. vol. 72 (1960) pp. 458-520, and all references (like 6.1 below) are to that paper.

Clearly (2) follows from (1), with $X=\partial T, S=T-Y$.

To prove (1) let $U, V, f, g, a, b, \xi, \eta, c_{1}, c_{2}, c_{3}$ be as in 6.1 with $\Gamma=A$, let

$$
\begin{aligned}
\epsilon & =\inf \{a /[3 n(\eta+1)], b /(3 n)\} \\
\Phi & =R^{n} \cap\{x: \text { distance }(x, A) \leqq 3 n \epsilon\}, \\
\Psi & =R^{n} \cap\{x: \text { distance }(x, B) \leqq 3 n \epsilon\},
\end{aligned}
$$

let $K$ be the subcomplex of $\mu_{\epsilon}\left(C^{\prime}\right)$ (see $2.1,5.1$ ) consisting of the cubes contained in $\Phi$, and let $\Omega$ be the group of those $k$ dimensional chains of $K$ which belong to

Presented to the Society, April 16, 1960; received by the editors December 2, 1960.

1 This work was supported in part by a Sloan Fellowship. 